\title{
Eigenvalues of Elliptic Systems for the Mixed Problem in Perturbations of Lipschitz Domains with Nonhomogeneous Neumann Boundary Conditions
}

\author{
MIYAZAKI Kohei and TAYLOR Justin L.* \\ Department of Mathematics $\mathcal{E}$ Statistics, Murray State University, Murray, \\ Kentucky, U.S.A.
}

Received 31 October 2019; Accepted 31 January 2020

\begin{abstract}
We study eigenvalues of an elliptic operator with mixed boundary conditions on very general decompositions of the boundary. We impose nonhomogeneous conditions on the part of the boundary where the Neumann term lies in a certain Sobolev or $L^{p}$ space. Our work compares the behavior of and gives a relationship between the eigenvalues and eigenfunctions on the unperturbed and perturbed domains, respectively.
\end{abstract}

AMS Subject Classifications: 35A23, 35B65, 35D30, 35J57, 35M32, 35P99

Chinese Library Classifications: O175.9, O175.25

Key Words: Eigenvalues; elliptic systems; mixed problem; perturbed domains.

\section{Introduction}

The literature contains much analysis on the study of eigenvalues for elliptic equations, but work on systems of equations is much more scarce. Many of the estimates used for equations do not hold for systems and thus analysis for systems requires something different. Moreover, we use the reverse Hölder technique frequently to achieve our estimates. In this paper, we look at the behavior of eigenvalues and eigenfunctions on perturbed domains and compare them to ones on the unperturbed domain. We give a simple characterization of families of perturbed domains, which include dumbbell shaped domains, but these families may be quite general. We work in Lipschitz domains and assume that

*Corresponding author. Email addresses: kmiyazaki@murraystate.edu (K. Miyazaki), jtaylor52@murraysta -te.edu (J. L. Taylor) 
the coefficients of the operator are bounded and symmetric. Furthermore, we assume that the Dirichlet set $D$ satisfies a corkscrew condition, which allows for a rather general decomposition of the boundary. A term which lies in a Sobolev space or $L^{p}$ space is imposed on the Neumann set $N$.

There are many results on the study of eigenvalues for equations on perturbed domains when we have Dirichlet boundary conditions. A classic paper by Babuska and Výborný [1] shows continuity of Dirichlet eigenvalues for elliptic equations under a regular variation of the domain. Work by Davies [2] and Pang [3] studies the relationship between Dirichlet eigenvalues and corresponding eigenfunctions in a domain $\Omega$ and eigenvalues and eigenfunctions in sets of the form $R(\varepsilon)=\{x \in \Omega: \operatorname{dist}(x, \partial \Omega) \geq \varepsilon\}$. Two papers by Chavel and Feldman [4] and Anné and Colbois [5] examine eigenvalues on compact manifolds with a small handle and Dirichlet conditions on the ends of the handle. More recent work for Dirichlet conditions includes work by Daners [6], which shows convergence of solutions to elliptic equations on sequences of domains. Burenkov and Lamberti [7] prove spectral estimates for higher-order elliptic operators on domains in certain Hölder classes. Kozlov [8] gives asymptotics of Dirichlet eigenvalues for domains in $\mathbb{R}^{n}$ and Grieser and Jerison [9] also give asymptotics for Dirichlet eigenvalues and eigenfunctions on plane domains.

When a Neumann condition is placed on part of the boundary, the eigenvalue problem is much more difficult to analyze. A classic example by Courant and Hilbert [10] shows that continuity of eigenvalues is not generally obtained for Neumann eigenvalues if the domain is only $C^{0}$. In fact, Arrieta, Hale, and Han [11] show that if the domain is not sufficiently smooth, none of the Neumann eigenvalues $\left\{\lambda_{m}^{\varepsilon}\right\}$ converge for $m \geq 3$. However, if one places more regularity on the domains, rates of convergence are achievable. This is illustrated in work by Jimbo [12], Jimbo and Kosugi [13], and Brown, Hislop, and Martinez [14].

As mentioned earlier, there seems to be a lot less work on the study of perturbed domains with systems of equations. Fang [15] studied the behavior of the second eigenvalue in a perturbed domain for a system of equations in $\mathbb{R}^{2}$. Taylor [16] provided rates of convergence for Dirichlet eigenvalues involving elliptic systems on domains with low regularity. More recent work by Collins and Taylor [17] showed convergence of eigenvalues for the mixed problem with homogeneous Dirichlet and Neumann boundary conditions. The contribution in this paper continues the study of eigenvalues for these types of operators for the mixed problem when the Neumann term is nontrivial.

\section{Preliminaries and main results}

We will be working on a bounded Lipschitz domain $\Omega$. This means that locally on the boundary, $\Omega$ is a domain which lies above the graph of a Lipschitz function. In order to give a formal definition, we introduce coordinate cylinders. Given a constant $M>0$, $x \in \partial \Omega$, and $r>0$, we define a coordinate cylinder $Z_{r}(x)=\left\{y:\left|y^{\prime}-x^{\prime}\right|<r,\left|y_{n}-x_{n}\right|<\right.$ 
$(2+4 M) r\}$. Here, we use coordinates $\left(x^{\prime}, x_{n}\right) \in \mathbb{R}^{n-1} \times \mathbb{R}$ and assume that this coordinate system is a translation and rotation of the standard coordinates. $\Omega$ is said to be a Lipschitz domain if for each $x \in \partial \Omega$, there exists a coordinate cylinder $Z_{r}(x)$ and a Lipschitz function $\phi: \mathbb{R}^{n-1} \rightarrow \mathbb{R}$ with Lipschitz constant $M$ so that

$$
\begin{aligned}
& \Omega \cap Z_{r}(x)=\left\{\left(y^{\prime}, y_{n}\right): y_{n}>\phi\left(y^{\prime}\right)\right\} \cap Z_{r}(x), \\
& \partial \Omega \cap Z_{r}(x)=\left\{\left(y^{\prime}, y_{n}\right): y_{n}=\phi\left(y^{\prime}\right)\right\} \cap Z_{r}(x) .
\end{aligned}
$$

Since the boundary $\partial \Omega$ is compact, there exists a small constant $r_{0}$ so that we may cover it with a finite collection of coordinate cylinders $\left\{Z_{r_{0}}\left(x_{i}\right)\right\}_{i=1}^{N}$ and so that for each $i$, $Z_{100 r_{0} \sqrt{1+M^{2}}}\left(x_{i}\right)$ is also a coordinate cylinder.

We define our local domains as follows: For $x \in \partial \Omega$ and $r \in\left(0,100 r_{0}\right)$, we define surface cylinders $\Delta_{r}(x)=Z_{r}(x) \cap \partial \Omega$. When $x \in \Omega$ and $r \in\left(0,100 r_{0}\right)$, if $\operatorname{dist}(x, \partial \Omega)>r$, then $S_{r}(x)=$ $B_{r}(x)$ and $\Delta_{r}(x)=\varnothing$. Otherwise, if $\operatorname{dist}(x, \partial \Omega) \leq r$, then from the definition of a coordinate cylinder, $x$ lies in one of the cylinders $Z_{100 r_{0} \sqrt{1+M^{2}}}\left(x_{i}\right)$. If $x=\left(x^{\prime}, x_{n}\right)$ in the coordinate system for this cylinder, then we put $\hat{x}=\left(x^{\prime}, \phi\left(x^{\prime}\right)\right)$ and define $S_{r}(x)=Z_{r}(\hat{x}) \cap \Omega$ and $\Delta_{r}(x)=Z_{r}(\hat{x}) \cap \partial \Omega$. We note that with our choice of $r_{0}$ and definitions of cylinders, our local domains are star-shaped domains which will allow us to use Sobolev and Korn inequalities.

We let $\mathscr{D}_{1}$ and $\mathscr{D}_{2}$ be two nonempty, open, disjoint, connected, and bounded Lipschitz domains in $\mathbb{R}^{n}$ and form the domain $\Omega_{0}=\mathscr{D}_{1} \cup \mathscr{D}_{2}$. Decompose the boundary $\partial \Omega_{0}=D \cup N$ and $D \cap N=\varnothing$ where $D$ is nonempty and relatively open with respect to $\partial \Omega_{0}$. We assume the corkscrew condition on $D$. To define this condition, let $\Lambda$ denote the boundary of $D$ in $\partial \Omega_{0}$.

Definition 2.1. We say that $D$ satisfies the corkscrew condition if for all $x \in \Lambda$ and $r \in\left(0,100 r_{0}\right)$, there exists $x_{r} \in D$ so that $\left|x_{r}-x\right|<r$ and dist $\left(x_{r}, \Lambda\right)>M^{-1}$.

The corkscrew condition allows for a quite general decomposition of the boundary. It follows that if $D$ satisfies the corkscrew condition, then we have the following lemma shown in Taylor et al. [18]:

Lemma 2.1. If $x \in D$ and $r \in\left(0,100 r_{0}\right)$, then there exists $x_{r} \in D$ and a constant $c=c(M)$, only depending on $M$, so that $\left|x-x_{r}\right|<r$ and $\Delta_{c r}\left(x_{r}\right) \subset D$. Furthermore,

$$
\sigma\left(\Delta_{r}(x) \cap D\right) \geq c r^{n-1} .
$$

In the above lemma, $\sigma$ denotes the surface measure. We again note that the local domains mentioned earlier are star-shaped domains. Thus with the aid of Lemma 2.1, we may apply Sobolev and Poincaré estimates later.

We define the family of perturbations for a suitable $\varepsilon_{0},\left\{T_{\varepsilon}\right\}_{0<\varepsilon<\varepsilon_{0}}$ to be a family of open sets with Lipschitz boundary such that

$$
T_{\widetilde{\varepsilon}} \subset T_{\varepsilon} \text { if } \widetilde{\varepsilon} \leq \varepsilon
$$


and if $\left|T_{\varepsilon}\right|$ denotes the $\mathbb{R}^{n}$ Lebesgue measure of $T_{\varepsilon}$, then

$$
\left|T_{\varepsilon}\right| \leq C \varepsilon^{d}
$$

where $C$ and $0<d \leq n$ are independent of $\varepsilon$. This allows the perturbations to be quite general. We fix two points $p_{1} \in \partial \mathscr{D}_{1}, p_{2} \in \partial \mathscr{D}_{2}$, and define $\Delta_{\varepsilon}^{1}=Z_{\varepsilon}\left(p_{1}\right) \cap \partial \Omega_{0}, \Delta_{\varepsilon}^{2}=Z_{\varepsilon}\left(p_{2}\right) \cap$ $\partial \Omega_{0}$. We require the connections from $T_{\varepsilon}$ to $\mathscr{D}_{1}$ and $\mathscr{D}_{2}$ to be contained in $\Delta_{\varepsilon}^{1}$ and $\Delta_{\varepsilon}^{2}$, respectively. That is, $T_{\varepsilon} \cap \mathscr{D}_{1}=\varnothing$ and $\overline{T_{\varepsilon}} \cap \overline{\mathscr{D}_{1}} \subset \Delta_{\varepsilon}^{1}$. We also require that for each $\varepsilon<$ $\varepsilon_{0}$, we have $\Delta_{\varepsilon}^{1} \cap D \neq \varnothing$. In other words, the connection is always touching $D$ in some sense, but may also touch $N$. We define a similar condition for $\mathscr{D}_{2}$ and $\Delta_{\varepsilon}^{2}$. Then for any $\varepsilon$, define $\Omega_{\varepsilon}$ to be the interior of the set $\overline{\Omega_{0}} \cup \overline{T_{\varepsilon}}$. So, you may think of $T_{\varepsilon}$ as a "tube" connecting each of the two domains. We now have the family of domains $\left\{\Omega_{\varepsilon}\right\}_{0 \leq \varepsilon<\varepsilon_{0}}$. Let $\widetilde{D}=\partial \Omega_{\varepsilon} \cap\left[\partial T_{\varepsilon} \cup D\right]$ and $\widetilde{N}=\partial \Omega_{\varepsilon} \cap N$. We let $\left(W^{1, p}\left(\Omega_{\varepsilon}\right)\right)^{m}=W^{1, p}\left(\Omega_{\varepsilon}\right) \times \ldots \times W^{1, p}\left(\Omega_{\varepsilon}\right)$ be the vector-valued Sobolev space taking values in $\mathbb{R}^{m}$ and define $\left(W_{\widetilde{D}}^{1, p}\left(\Omega_{\varepsilon}\right)\right)^{m}$ to be the closure of $\left(W^{1, p}\left(\Omega_{\varepsilon}\right)\right)^{m}$ in $\left\{u \in\left(C^{\infty}\left(\overline{\Omega_{\varepsilon}}\right)\right)^{m}: u=0\right.$ in a neighborhood of $\left.\widetilde{D}\right\}$. We also define

$$
\left(L^{p}\left(\partial \Omega_{\varepsilon}\right)\right)^{m}=L^{p}\left(\partial \Omega_{\varepsilon}\right) \times \ldots \times L^{p}\left(\partial \Omega_{\varepsilon}\right),\left(W_{\widetilde{D}}^{1 / 2, p}\left(\partial \Omega_{\varepsilon}\right)\right)^{m}
$$

to be the image of $\left(W_{\widetilde{D}}^{1, p}\left(\Omega_{\varepsilon}\right)\right)^{m}$ under the trace map, and $\left(W_{\widetilde{D}}^{-1 / 2, p^{\prime}}\left(\partial \Omega_{\varepsilon}\right)\right)^{m}$ to be the dual of $\left(W_{\widetilde{D}}^{1 / 2, p}\left(\partial \Omega_{\varepsilon}\right)\right)^{m}$. Since we are dealing with vector-valued functions $u \in\left(W^{1, p}\left(\Omega_{\varepsilon}\right)\right)^{m}$, the gradient $\nabla u$ should be interpreted as a matrix $B_{i j}=\frac{\partial}{\partial x_{j}} u^{i}$ and $|\nabla u|$ is the Frobenius norm of $\nabla u$.

Since $D$ satisfies the corkscrew condition for any $\varepsilon$, and $\Omega_{\varepsilon}$ is a bounded Lipschitz Domain, Lemma 2.1 implies the Poincaré inequality for any $1 \leq p<\infty$,

$$
\int_{\Omega_{\varepsilon}}|u|^{p} \mathrm{~d} y \leq C \int_{\Omega_{\varepsilon}}|\nabla u|^{p} \mathrm{~d} y, \quad u \in\left(W_{\widetilde{D}}^{1, p}\left(\Omega_{\varepsilon}\right)\right)^{m} .
$$

Consequently, we have the trace inequality as given in Cianchi et al. [19],

$$
\int_{\partial \Omega_{\varepsilon}}|u|^{p} \mathrm{~d} y \leq C \int_{\Omega_{\varepsilon}}|\nabla u|^{p} \mathrm{~d} y, \quad u \in\left(W_{\widetilde{D}}^{1, p}\left(\Omega_{\varepsilon}\right)\right)^{m} .
$$

Define

$$
\bar{u}_{x, r}:= \begin{cases}0, & \text { if } \operatorname{dist}\left(S_{r}(x), D\right)=0 \\ f_{S_{r}(x)} u(y) \mathrm{d} y, & \text { if } \operatorname{dist}\left(S_{r}(x), D\right)>0 .\end{cases}
$$

We also have Sobolev-Poincaré inequalities for our local domains, taken from Ott and Brown [20].

Lemma 2.2. Suppose $1 \leq p<n$ and $\frac{1}{q}=\frac{1}{p}-\frac{1}{n}$. Then we have

$$
\left(\int_{S_{r}(x)}\left|u-\bar{u}_{x, r}\right|^{q} \mathrm{~d} y\right)^{1 / q} \leq C\left(\int_{S_{2 r}(x)}|\nabla u|^{p} \mathrm{~d} y\right)^{1 / p}, \quad u \in\left(W_{\widetilde{D}}^{1, p}\left(\Omega_{\varepsilon}\right)\right)^{m} .
$$


Lemma 2.3. Let $1 \leq p<n$ and choose $q$ so that $\frac{1}{q}=\frac{1}{p}-\left(1-\frac{1}{p}\right)\left(\frac{1}{n-1}\right)$. Then we have

$$
\left(\int_{\Delta_{r}(x)}\left|u-\bar{u}_{x, r}\right|^{q} \mathrm{~d} \sigma(y)\right)^{1 / q} \leq C\left(\int_{S_{2 r}(x)}|\nabla u|^{p} \mathrm{~d} y\right)^{1 / p}, u \in\left(W_{\widetilde{D}}^{1, p}\left(\Omega_{\varepsilon}\right)\right)^{m} .
$$

The previous lemmas hold with $S_{r}(x)$ as the domain of integration on the right side if $\operatorname{dist}\left(S_{r}(x), D\right)>0$. Otherwise, we must expand $S_{r}(x)$ in order to use the corkscrew condition. The inequalities (2.5) and (2.6) imply the Poincaré inequalities for any $1 \leq p<\infty$,

$$
\begin{array}{ll}
\left(\int_{S_{r}(x)}\left|u-\bar{u}_{x, r}\right|^{p} \mathrm{~d} y\right)^{1 / p} \leq \operatorname{Cr}\left(\int_{S_{2 r}(x)}|\nabla u|^{p} \mathrm{~d} y\right)^{1 / p}, \quad u \in\left(W_{\widetilde{D}}^{1, p}\left(\Omega_{\varepsilon}\right)\right)^{m}, \\
\left(\int_{\Delta_{r}(x)}\left|u-\bar{u}_{x, r}\right|^{p} \mathrm{~d} \sigma(y)\right)^{1 / p} \leq \operatorname{Cr} \frac{p-1}{p}\left(\int_{S_{2 r}(x)}|\nabla u|^{p} \mathrm{~d} y\right)^{1 / p}, \quad u \in\left(W_{\widetilde{D}}^{1, p}\left(\Omega_{\varepsilon}\right)\right)^{m} .
\end{array}
$$

Now we define the eigenvalue problem. Let $L=-\frac{\partial}{\partial x_{j}}\left(A_{i j} \frac{\partial}{\partial x_{i}}\right)$ where the $A_{i j}$ are coefficient $m \times m$ matrices which have measurable and bounded entries, $a_{i j}^{\alpha \beta}$ which also satisfy the symmetry condition $a_{i j}^{\alpha \beta}=a_{j i}^{\beta \alpha}$ for $i, j=1, \ldots, n$ and $\alpha, \beta=1, \ldots, m$. We consider the mixed eigenvalue problem

$$
\begin{cases}L u=\lambda u, & \text { in } \Omega_{\mathcal{\varepsilon}}, \\ u=0, & \text { on } \widetilde{D}, \\ A_{i j} \frac{\partial u}{\partial x_{i}} \nu^{j}=f_{N}, & \text { on } \widetilde{N},\end{cases}
$$

where $f_{N} \in\left(W_{D}^{-1 / 2, p^{\prime}}\left(\partial \Omega_{0}\right)\right)^{m} \cap\left(W_{\widetilde{D}}^{-1 / 2, p^{\prime}}\left(\partial \Omega_{\varepsilon}\right)\right)^{m}$ for $p^{\prime}>2$ and $v$ is the outward unit normal vector. $L$ may also be viewed in coordinate form

$$
(L u)^{\beta}=-\sum_{\alpha=1}^{m} \sum_{i, j=1}^{n} \frac{\partial}{\partial x_{j}}\left(a_{i j}^{\alpha \beta} \frac{\partial u^{\alpha}}{\partial x_{i}}\right), \quad \beta=1, \ldots, m .
$$

Above in (2.9) and throughout the rest of this paper we use the convention of summing over repeated indices, where $i$ and $j$ will sum from 1 to $n$ and $\alpha, \beta$, and $\gamma$ will sum from 1 to $m$. Letting $u_{j}^{\alpha}:=\frac{\partial u^{\alpha}}{\partial x_{j}}$, define the bilinear form on $\left(W_{\widetilde{D}}^{1,2}\left(\Omega_{\varepsilon}\right)\right)^{m} \times\left(W_{\widetilde{D}}^{1,2}\left(\Omega_{\varepsilon}\right)\right)^{m}$,

$$
B_{\varepsilon}(u, v):=\int_{\Omega_{\varepsilon}} a_{i j}^{\alpha \beta}(x) u_{i}^{\alpha}(x) v_{j}^{\beta}(x) \mathrm{d} x .
$$

We say that the number $\lambda$ is an eigenvalue of $L$ with eigenfunction $u \in\left(W_{\widetilde{D}}^{1,2}\left(\Omega_{\varepsilon}\right)\right)^{m}$, if $u \neq \equiv$ and

$$
B_{\varepsilon}(u, v)=\lambda \int_{\Omega_{\varepsilon}} u^{\gamma}(x) v^{\gamma}(x) \mathrm{d} x+\left\langle f_{N}, v\right\rangle_{\partial \Omega_{\varepsilon}} \quad \text { for any } v \in\left(W_{\widetilde{D}}^{1,2}\left(\Omega_{\varepsilon}\right)\right)^{m},
$$


where $\langle\cdot, \cdot\rangle_{\partial \Omega_{\varepsilon}}$ denotes the pairing of duality on $\left(W_{\widetilde{D}}^{-1 / 2, p^{\prime}}\left(\partial \Omega_{\varepsilon}\right)\right)^{m} \times\left(W_{\widetilde{D}}^{1 / 2, p}\left(\partial \Omega_{\varepsilon}\right)\right)^{m}$. We define the Rayleigh quotient $R_{\varepsilon}$ on $\left(W^{1,2}\left(\Omega_{\varepsilon}\right)\right)^{m}$ as

$$
R_{\varepsilon}(u):=\frac{B_{\varepsilon}(u, u)-\left\langle f_{N}, u\right\rangle_{\partial \Omega_{\varepsilon}}}{\|u\|_{L^{2}\left(\Omega_{\varepsilon}\right)}^{2}}
$$

for $u \neq \equiv 0$. It easily follows that if $\lambda$ is an eigenvalue with eigenfunction $u$, then

$$
\lambda=R_{\varepsilon}(u) .
$$

We point out that if $f_{N}$ is a function on $N$, then we may identify it with an element of $\left(W_{\widetilde{D}}^{-1 / 2, p^{\prime}}\left(\partial \Omega_{\varepsilon}\right)\right)^{m}$ by

$$
\left\langle f_{N}, v\right\rangle_{\partial \Omega_{\varepsilon}}=\int_{\widetilde{N}} f_{N} v \mathrm{~d} \sigma \quad \text { for any } v \in\left(W_{\widetilde{D}}^{1 / 2, p}\left(\partial \Omega_{\varepsilon}\right)\right)^{m} .
$$

Sobolev trace embedding implies $\left(W_{\widetilde{D}}^{1 / 2,2}\left(\partial \Omega_{\varepsilon}\right)\right)^{m} \subset\left(L^{q}\left(\partial \Omega_{\varepsilon}\right)\right)^{m}$ for $q=\frac{2(n-1)}{n-2}$ when $n \geq 3$ or $1 \leq q<\infty$ when $n=2$. Thus for any test function $v$ from (2.10), the integral on the right side of (2.12) is finite if $f_{N} \in\left(L^{\frac{2(n-1)}{n}}\left(\partial \Omega_{\varepsilon}\right)\right)^{m}$ when $n \geq 3$ or $f_{N} \in\left(L^{q}\left(\partial \Omega_{\varepsilon}\right)\right)^{m}$ for any $q>1$ when $n=2$. Therefore, the weak formulation is properly defined when $f_{N}$ is a function in the space $\left(L^{p^{\prime}}\left(\partial \Omega_{\varepsilon}\right)\right)^{m}$ for $p^{\prime}>2$.

Throughout this paper, we will assume that for any $u \in\left(W_{\widetilde{D}}^{1,2}\left(\Omega_{\varepsilon}\right)\right)^{m}$, we have Gårding's inequality

$$
C_{1} \int_{\Omega_{\varepsilon}}|\nabla u|^{2} \mathrm{~d} x \leq \int_{\Omega_{\varepsilon}} a_{i j}^{\alpha \beta}(x) u_{i}^{\alpha}(x) u_{j}^{\beta}(x) \mathrm{d} x+C_{2} \int_{\Omega_{\varepsilon}}|u|^{2} \mathrm{~d} x .
$$

This inequality will be true if $L$ satisfies certain ellipticity conditions and if we assume certain smoothness conditions on the coefficients, such as uniform boundedness. In particular, we say that $L$ satisfies a strong Legendre condition or a strong ellipticity condition if there exists $\theta>0$ so that

$$
a_{i j}^{\alpha \beta}(x) \xi_{i}^{\alpha} \xi_{j}^{\beta} \geq \theta|\xi|^{2}, \quad \xi \in \mathbb{R}^{m \times n}, \text { a.e. } x \in \Omega_{\varepsilon} .
$$

If $L$ satisfies (2.14), then it is clear that Gårding's inequality holds for any $u \in\left(W_{\widetilde{D}}^{1,2}\left(\Omega_{\varepsilon}\right)\right)^{m}$. We also say that $L$ satisfies the Legendre-Hadamard condition if there exists $\theta>0$ so that

$$
a_{i j}^{\alpha \beta}(x) \xi^{\alpha} \xi^{\beta} \psi^{i} \psi^{j} \geq \theta|\xi|^{2}|\psi|^{2}, \quad \xi \in \mathbb{R}^{m}, \quad \psi \in \mathbb{R}^{n} \text {, a.e. } x \in \Omega_{\varepsilon} .
$$

It is well-known that if $L$ satisfies the Legendre-Hadamard condition with continuous coefficients in $\bar{\Omega}$, then Gårding's inequality holds for any $u \in\left(W_{\widetilde{D}}^{1,2}\left(\Omega_{\varepsilon}\right)\right)^{m}$. Please see Treves [21] for details.

The Lamé system is defined as $L u=-\operatorname{div} \zeta(u)$, where $\zeta(u)$ denotes the stress tensor

$$
\zeta_{j}^{\beta}(u):=a_{i j}^{\alpha \beta} u_{i}^{\alpha} .
$$


Here, the Lamé moduli $v(x)$ and $\mu(x)$ are given by

$$
a_{i j}^{\alpha \beta}(x)=v(x) \delta_{i \alpha} \delta_{j \beta}+\mu(x) \delta_{i j} \delta_{\alpha \beta}+\mu(x) \delta_{i \beta} \delta_{j \alpha},
$$

where $\delta_{i j}$ is the Kronecker delta. The functions $v(x)$ and $\mu(x)$ are both assumed to be bounded and measurable and satisfy the conditions

$$
v(x) \geq 0 \quad \mu(x) \geq b>0 .
$$

Using Korn's 1st Inequality as given in [22], it is easy to see that for the Lamé system, Gårding's inequality is satisfied for all $u \in\left(W_{\widetilde{D}}^{1,2}\left(\Omega_{\varepsilon}\right)\right)^{m}$.

The aim of this paper is to relate eigenvalues and eigenfunctions on the unperturbed domain to eigenvalues and approximated eigenfunctions on the perturbed domain. In particular, we show that if an eigenfunction is in a certain class, the relationship to its corresponding eigenvalue on $\Omega_{\varepsilon}$ is similar to the relationship between an eigenfunction and corresponding eigenvalue on $\Omega_{0}$. That is, an eigenvalue on $\Omega_{\varepsilon}$ acts as an eigenvalue on $\Omega_{0}$ and the approximated eigenfunction on $\Omega_{\varepsilon}$ acts as an eigenfunction on $\Omega_{0}$. These relationships are illustrated in the main results of our work, namely Theorems 4.1 and 4.2 .

\section{A reverse Hölder estimate}

A key ingredient in our work will involve the reverse Hölder argument introduced by Gehring [23] and refined by Giaquinta and Modica [24,25]. The proof of the following Caccioppoli estimate is similar to the one given in Collins and Taylor [17], but some modifications are needed since we have a boundary term. For any $f \in\left(L_{\text {loc }}^{1}(\partial \Omega)\right)^{m}$ and $r>0$, define a local version of the Hardy-Littlewood maximal function $P_{r} f: \bar{\Omega} \rightarrow \mathbb{R}$ by

$$
P_{r} f(x):=\sup _{r>s>0} f_{\Delta_{s}(x)}|f(y)| \mathrm{d} \sigma(y) .
$$

Theorem 3.1. Let $u$ be an eigenfunction with eigenvalue $\lambda$ associated to the operator $L$. If $f_{N}$ is a function in $\left(L^{2}\left(\partial \Omega_{\varepsilon}\right)\right)^{m}$, there exists $\widetilde{r}$ such that when $\widetilde{r} \geq r>0, x \in \Omega_{\varepsilon}$, and $S_{r}=S_{r}(x)$, we have

$$
\begin{array}{r}
f_{S_{r}(x)}|\nabla u|^{2} \mathrm{~d} y \leq C\left(f_{S_{4 r}(x)}|\nabla u|^{\frac{2 n}{n+2}} \mathrm{~d} y\right)^{\frac{n+2}{n}}+C|\lambda| f_{S_{4 r}(x)} u^{2} \mathrm{~d} y \\
+C f_{S_{4 r}(x)} P_{4 r} f_{N}^{2} \mathrm{~d} y+\chi f_{S_{4 r}(x)}|\nabla u|^{2} \mathrm{~d} y
\end{array}
$$

Here, $\chi$ can be chosen to be any number less than one and $C>0$ depends on $\max _{i, j, \alpha, \beta}\left\|a_{i j}^{\alpha \beta}\right\|_{L^{\infty}\left(\Omega_{\varepsilon}\right)}, n$, $m, M$, and the constants from (2.13). Moreover, $\widetilde{r}$ depends on the choice of $\chi$ and the coefficients $a_{i j}^{\alpha \beta}$. 
Proof. Since there are 3 ellipticity conditions, there are 3 cases to consider. We note that in Collins and Taylor [17, Theorem 3.1], all cases are proven with the exception of the boundary term. Following along their proof by using the same procedure, for any fixed constant $\chi<\frac{1}{2}$, we may arrive at the inequality

$$
\begin{aligned}
\int_{S_{r}}|\nabla u|^{2} \mathrm{~d} y \leq \frac{C}{r^{2}} & \int_{S_{2 r}}\left|u-\bar{u}_{x, 2 r}\right|^{2} \mathrm{~d} y+C|\lambda| \int_{S_{2 r}}|u|^{2} \mathrm{~d} y+\chi \int_{S_{2 r}}|\nabla u|^{2} \mathrm{~d} y \\
& +C \int_{\Delta_{2 r}}\left|f_{N}\right|\left|u-\bar{u}_{x, 2 r}\right| \mathrm{d} \sigma(y) .
\end{aligned}
$$

To deal with the boundary term, we choose any $\rho>0$ so that

$$
C \int_{\Delta_{2 r}}\left|f_{N}\right|\left|u-\bar{u}_{x, 2 r}\right| \mathrm{d} \sigma(y) \leq C \rho \int_{\Delta_{2 r}}\left|f_{N}\right|^{2} \mathrm{~d} \sigma(y)+\frac{C}{\rho} \int_{\Delta_{2 r}}\left|u-\bar{u}_{x, 2 r}\right|^{2} \mathrm{~d} \sigma(y) .
$$

Next, apply the Poincaré inequality (2.8) to get

$$
\frac{C}{\rho} \int_{\Delta_{2 r}}\left|u-\bar{u}_{x, 2 r}\right|^{2} \mathrm{~d} \sigma(y) \leq \frac{C r}{\rho}\left(\int_{S_{4 r}}|\nabla u|^{2} \mathrm{~d} y\right) .
$$

Now divide both sides of (3.3) by $\left|S_{r}\right|$ and use (3.4) to obtain

$$
\frac{C}{\left|S_{r}\right|} \int_{\Delta_{2 r}}\left|f_{N}\right|\left|u-\bar{u}_{x, 2 r}\right| \mathrm{d} \sigma(y) \leq \frac{C \rho}{\left|S_{r}\right|} \int_{\Delta_{2 r}}\left|f_{N}\right|^{2} \mathrm{~d} \sigma(y)+\frac{C r}{\rho\left|S_{4 r}\right|} \int_{S_{4 r}(x)}|\nabla u|^{2} \mathrm{~d} y .
$$

If we now choose $\rho=\frac{C r}{\chi}$ we have that

$$
\begin{aligned}
& \frac{C}{\left|S_{r}\right|} \int_{\Delta_{2 r}}\left|f_{N}\right|\left|u-\bar{u}_{x, 2 r}\right| \mathrm{d} \sigma(y) \leq C f_{\Delta_{2 r}}\left|f_{N}\right|^{2} \mathrm{~d} \sigma(y)+\chi f_{S_{4 r}}|\nabla u|^{2} \mathrm{~d} y \\
& \leq C P_{2 r} f_{N}^{2}(x)+\chi f_{S_{4 r}}|\nabla u|^{2} \mathrm{~d} y \leq C f_{S_{2 r}} P_{4 r} f_{N}^{2} \mathrm{~d} y+\chi f_{S_{4 r}}|\nabla u|^{2} \mathrm{~d} y,
\end{aligned}
$$

where we have used [26, Lemma A.2.] on the last line. Now if we divide (3.2) by $\left|S_{r}\right|$, apply the Sobolev-Poincare inequality (2.5) to the first term on the right side, and use (3.6), we obtain the result (3.1).

The next lemma taken from Ott and Brown [27] gives an $L^{q}$ estimate on $P_{r} f$.

Lemma 3.1. Let $p>1$ and choose $q$ so that $1 \leq q \leq \frac{p n}{n-1}$. If $f \in\left(L_{l o c}^{p}\left(\partial \Omega_{\varepsilon}\right)\right)^{m}$, then for any $x \in \partial \Omega$ and any $0<r<r_{0}$, we have

$$
\left(f_{S_{r}(x)}\left|P_{r} f\right|^{q} \mathrm{~d} y\right)^{\frac{1}{q}} \leq C\left(f_{\Delta_{2 r}(x)}|f|^{p} \mathrm{~d} \sigma\right)^{\frac{1}{p}},
$$

where $C$ is a constant only depending on $M$ and the dimension $n$. 
To prove the reverse Hölder estimate given in Theorem 3.3, we introduce the maximal functions below and a theorem taken from [24].

Definition 3.1. Given $f \in\left(L_{l o c}^{1}\left(\mathbb{R}^{n}\right)\right)^{m}$ and a number $R$, we define the Hardy-Littlewood maximal functions by

$$
\begin{aligned}
& M(f)(x):=\sup _{r>0} f_{B_{r}(x)}|f(y)| \mathrm{d} y, \\
& M_{R}(f)(x):=\sup _{R>r>0} f_{B_{r}(x)}|f(y)| \mathrm{d} y .
\end{aligned}
$$

Theorem 3.2. Let $r>q>1$, and $Q_{R}$ be a cube in $\mathbb{R}^{n}$ with sidelength $R$ centered at 0 . Also, define $d(x)=\operatorname{dist}\left(x, \partial Q_{R}\right)$. If $f$ and $g$ are measurable functions such that $f \in\left(L^{r}\left(Q_{R}\right)\right)^{m}, g \in$ $\left(L^{q}\left(Q_{R}\right)\right)^{m}, f=g=0$ outside $Q_{R}$, and with the added condition that

$$
M_{\frac{d(x)}{2}}\left(|g|^{q}\right)(x) \leq b M^{q}(g)(x)+M\left(|f|^{q}\right)+a M\left(|g|^{q}\right)(x)
$$

for almost every $x$ in $Q_{R}$ where $b \geq 0$ and $0 \leq a<1$, then $g \in\left(L^{p}\left(Q_{\frac{R}{2}}\right)\right)^{m}$, for $p \in[q, q+\epsilon)$ and

$$
\left(f_{Q_{R / 2}}|g|^{p}(y) \mathrm{d} y\right)^{\frac{1}{p}} \leq C\left[\left(f_{Q_{R}}|g|^{q}(y) \mathrm{d} y\right)^{\frac{1}{q}}+\left(f_{Q_{R}}|f|^{p}(y) \mathrm{d} y\right)^{\frac{1}{p}}\right]
$$

where $\epsilon$ and $C$ depend on $b, q, n, a$ and $r$.

We now state and prove the main theorem in this section.

Theorem 3.3. Let $u$ be an eigenfunction with eigenvalue $\lambda$ associated to the operator L. If $f_{N}$ is a function in $\left(L^{p^{\prime}}\left(\partial \Omega_{\varepsilon}\right)\right)^{m}$ for $p^{\prime}>2$ with $f_{N}=0$ on $\widetilde{D}$, then there exists $\widetilde{p}>2$ so that

$$
\left(f_{\Omega_{\varepsilon}}|\nabla u|^{\widetilde{p}} \mathrm{~d} y\right) \leq C\left[\left(f_{\Omega_{\varepsilon}}|\nabla u|^{2} \mathrm{~d} y\right)^{\frac{\widetilde{p}}{2}}+|\lambda|^{\frac{\tilde{p}}{2}}\left(f_{\Omega_{\varepsilon}}|u|^{\widetilde{p}} \mathrm{~d} y\right)\right]+C\left(f_{\widetilde{N}}\left|f_{N}\right|^{\widetilde{p}} \mathrm{~d} \sigma\right) .
$$

Here, $\widetilde{p}$ and $C$ depend on $\max _{i, j, \alpha, \beta}\left\|a_{i j}^{\alpha \beta}\right\|_{L^{\infty}\left(\Omega_{\varepsilon}\right)}, n, m, M$, and the constants from (2.13). Moreover, $\widetilde{p}$ and $C$ are independent of $\varepsilon$.

Proof. Now if $u$ is an eigenfunction with eigenvalue $\lambda$, we have $u \in\left(W_{\widetilde{D}}^{1,2}\left(\Omega_{\varepsilon}\right)\right)^{m}$, and thus we may employ the Sobolev inequality to get that $|u| \in\left(L^{t}\left(\Omega_{\varepsilon}\right)\right)^{m}$ for some $t>2$. We choose a cube $Q_{R}$, centered at 0 , with sidelength $R$ such that $\Omega_{\varepsilon} \subset Q_{\frac{R}{2}}$, uniformly in E. Using (3.1) and (3.7) by setting $g=|\nabla u|^{\frac{2 n}{n+2}}, f=C^{\frac{n}{n+2}}\left(|\lambda||u|^{2}+\left(P_{4 r} f_{N}^{2}\right)\right)^{\frac{n}{n+2}}, q=\frac{n+2}{n}$, and $u=0$ outside $Q_{R}$, we obtain by a standard covering argument that

$$
\left(f_{\Omega_{\varepsilon}}|\nabla u|^{\frac{2 n p}{n+2}} \mathrm{~d} y\right)^{\frac{1}{p}}
$$




$$
\begin{aligned}
& \leq C\left[\left(f_{\Omega_{\varepsilon}}|\nabla u|^{2} \mathrm{~d} y\right)^{\frac{n}{n+2}}+\left(f_{\Omega_{\varepsilon}}\left(|\lambda||u|^{2}+\left(P_{4 r} f_{N}^{2}\right)\right)^{\frac{n p}{n+2}} \mathrm{~d} y\right)^{\frac{1}{p}}\right] \\
& \leq C\left[\left(f_{\Omega_{\varepsilon}}|\nabla u|^{2} \mathrm{~d} y\right)^{\frac{n}{n+2}}+|\lambda|^{\frac{n}{n+2}}\left(f_{\Omega_{\varepsilon}}|u|^{\frac{2 n p}{n+2}} \mathrm{~d} y\right)^{\frac{1}{p}}+\left(f_{\Omega_{\varepsilon}}\left(P_{4 r} f_{N}^{2}\right)^{\frac{n p}{n+2}} \mathrm{~d} y\right)^{\frac{1}{p}}\right] .
\end{aligned}
$$

Here, $\frac{n+2}{n} \leq p \leq \frac{n+2}{n}+\epsilon$, and $\epsilon$ from Theorem 3.2 is independent of $\varepsilon$ and any eigenvalue. Now setting $\widetilde{p}=\frac{2 n p}{n+2}$, and applying Lemma 3.1 with another covering argument, we have the result.

\section{Eigenvalue and eigenfunction analysis}

In this section, we will look at estimates on the eigenvalues and corresponding eigenfunctions. Because of the nonhomogeneity of the Neumann boundary condition, there is no reason to expect that we have a countable set of eigenvalues nor do we expect that each eigenfunction is bounded or can even be integrated. However, if we only look at eigenvalues contained in an interval which have corresponding eigenfunctions with bounded $L^{2}$ norms, then we can say a lot about the relationship between the eigenvalues and eigenfunctions on different perturbations. For the remainder of the paper we assume $f_{N}$ is a function in $\left(L^{p^{\prime}}\left(\partial \Omega_{\varepsilon}\right)\right)^{m}$ for $p^{\prime}>2$ with $f_{N}=0$ on $\widetilde{D}$. We define the following space:

Definition 4.1. Let $a, b \in \mathbb{R}$ such that $a<b$. Given $B>0$, we define $\mathscr{B}\left(a, b, B, \varepsilon_{0}\right)$ to be the space of functions $\phi \in\left(W_{\widetilde{D}}^{1,2}\left(\Omega_{\varepsilon}\right)\right)^{m}$ such that

$i$. There exists $\lambda \in[a, b]$ such that $L \phi=\lambda \phi$ in $\Omega_{\varepsilon}$ in the weak sense for some $\varepsilon \leq \varepsilon_{0}$.

ii. $\frac{1}{B} \leq \int_{\Omega_{\varepsilon}}|\phi(y)|^{2} \mathrm{~d} y \leq B$.

From this point, we denote an eigenvalue of $L$ with respect to $\Omega_{\varepsilon}$ as $\lambda^{\varepsilon}$ and $\phi_{\lambda}^{\varepsilon}$ as a corresponding eigenfunction. The next lemma gives a uniform bound on the $L^{\tilde{p}}$ norm of the gradient of an eigenfunction. The proof uses the reverse Hölder estimate (3.8).

Lemma 4.1. If $\phi_{\lambda}^{\varepsilon} \in \mathscr{B}\left(a, b, B, \varepsilon_{0}\right)$, then

$$
\left\|\nabla \phi_{\lambda}^{\varepsilon}\right\|_{L^{\widetilde{p}}\left(\Omega_{\varepsilon}\right)} \leq C .
$$

Here, $\widetilde{p}>2$ is from (3.8) and $C$ depends on $\left|\Omega_{0}\right|,\left\|f_{N}\right\|_{L^{\tilde{p}}(N)}, \max _{i, j, \alpha, \beta}\left\|a_{i j}^{\alpha \beta}\right\|_{L^{\infty}\left(\Omega_{\varepsilon}\right)}, n, m, M$, $\max \{|a|,|b|\}, B$, and the constants from (2.13). Moreover, $C$ is independent of $\varepsilon$.

Proof. The reverse Hölder estimate (3.8) implies

$$
\left(\int_{\Omega_{\varepsilon}}\left|\nabla \phi_{\lambda}^{\varepsilon}\right|^{\widetilde{p}} \mathrm{~d} y\right)
$$




$$
\leq C\left[\left(\int_{\Omega_{\varepsilon}}\left|\nabla \phi_{\lambda}^{\varepsilon}\right|^{2} \mathrm{~d} y\right)^{\frac{\tilde{p}}{2}}+\left|\lambda^{\varepsilon}\right|^{\tilde{p}}\left(\int_{\Omega_{\varepsilon}}\left|\phi_{\lambda}^{\varepsilon}\right|^{\widetilde{p}} \mathrm{~d} y\right)\right]+C\left(\int_{\widetilde{N}}\left|f_{N}\right|^{\widetilde{p}} \mathrm{~d} \sigma\right) .
$$

Also, Gårding's inequality (2.13) gives the estimate

$$
\begin{aligned}
C_{1} \int_{\Omega_{\varepsilon}}\left|\nabla \phi_{\lambda}^{\varepsilon}\right|^{2} \mathrm{~d} y & \leq \int_{\Omega_{\varepsilon}} a_{i j}^{\alpha \beta}\left(\phi_{\lambda}^{\varepsilon}\right)_{i}^{\alpha}\left(\phi_{\lambda}^{\varepsilon}\right)_{j}^{\beta} \mathrm{d} y+C_{2} \int_{\Omega_{\varepsilon}}\left|\phi_{\lambda}^{\varepsilon}\right|^{2} \mathrm{~d} y \\
& =\lambda^{\varepsilon} \int_{\Omega_{\varepsilon}}\left|\phi_{\lambda}^{\varepsilon}\right|^{2} \mathrm{~d} y+\left\langle f_{N}, \phi_{\lambda}^{\varepsilon}\right\rangle_{\partial \Omega_{\varepsilon}}+C_{2} \int_{\Omega_{\varepsilon}}\left|\phi_{\lambda}^{\varepsilon}\right|^{2} \mathrm{~d} y \\
& \leq\left(\lambda^{\varepsilon}+C_{2}\right) \int_{\Omega_{\varepsilon}}\left|\phi_{\lambda}^{\varepsilon}\right|^{2} \mathrm{~d} y+\frac{C}{\rho} \int_{\widetilde{N}}\left|f_{N}\right|^{2} \mathrm{~d} \sigma+\rho \int_{\partial \Omega_{\varepsilon}}\left|\phi_{\lambda}^{\varepsilon}\right|^{2} \mathrm{~d} \sigma,
\end{aligned}
$$

where $\rho>0$ is any constant. Therefore, applying the Poincaré estimate (2.4) on the last term and choosing $\rho$ appropriately, we obtain the estimate

$$
\int_{\Omega_{\varepsilon}}\left|\nabla \phi_{\lambda}^{\varepsilon}\right|^{2} \mathrm{~d} y \leq \frac{2}{C_{1}}\left[\left(\max \{|a|,|b|\}+C_{2}\right) B+C\left\|f_{N}\right\|_{L^{2}(\widetilde{N})}^{2}\right]=C .
$$

We next use the interpolation inequality

$$
\left(\int_{\Omega_{\varepsilon}}\left|\phi_{\lambda}^{\varepsilon}\right| \widetilde{p}^{\mathrm{p}} y\right)^{\frac{1}{\tilde{p}}} \leq\left(\int_{\Omega_{\varepsilon}}\left|\phi_{\lambda}^{\varepsilon}\right|^{q} \mathrm{~d} y\right)^{\frac{1-t}{q}}\left(\left.\int_{\Omega_{\varepsilon}}\left|\phi_{\lambda}^{\varepsilon}\right|\right|^{q^{*}} \mathrm{~d} y\right)^{\frac{t}{q^{*}}},
$$

where $t$ satisfies

$$
\frac{1}{\widetilde{p}}=\frac{1-t}{q}+\frac{t}{q^{*}}
$$

and $q^{*}$ is the Sobolev conjugate of $q \leq 2$. It follows from (4.3) and Sobolev's inequality that

$$
\left(\int_{\Omega_{\varepsilon}}\left|\phi_{\lambda}^{\varepsilon}\right| \widetilde{p} \mathrm{~d} y\right)^{\frac{1}{\tilde{p}}} \leq C .
$$

The inequalities (4.2), (4.3), and (4.5) now give the result.

Recall that the connections from $T_{\varepsilon}$ to $\Omega_{\varepsilon}$ are contained in $\Delta_{\varepsilon}^{1}=Z_{\varepsilon}\left(p_{1}\right) \cap \partial \Omega_{0}$ and $\Delta_{\varepsilon}^{2}=$ $Z_{\varepsilon}\left(p_{2}\right) \cap \partial \Omega_{0}$. Let $S_{\varepsilon}^{1}=Z_{\varepsilon}\left(p_{1}\right) \cap \Omega_{0}$ and $S_{\varepsilon}^{2}=Z_{\varepsilon}\left(p_{2}\right) \cap \Omega_{0}$. We define a cutoff function $\eta_{\varepsilon}$ : $\Omega_{\varepsilon} \rightarrow \mathbb{R}$ to be such that $0 \leq \eta \leq 1, \eta_{\varepsilon}=0$ in $\overline{T_{\varepsilon}}, \eta_{\varepsilon}=1$ outside $\overline{S_{\varepsilon}^{1}} \cup \overline{S_{\varepsilon}^{2}} \cup \overline{T_{\varepsilon}}$, and $\left|\nabla \eta_{\varepsilon}\right| \leq \frac{C}{\varepsilon}$. The next lemma states that the eigenfunction $\phi_{\lambda}^{\varepsilon}$ may be approximated by the function $\eta_{\varepsilon} \phi_{\lambda}^{\varepsilon}$ in $L^{2}$.

Lemma 4.2. If $\phi_{\lambda}^{\varepsilon} \in \mathscr{B}\left(a, b, B, \varepsilon_{0}\right)$, then

$$
\left\|\phi_{\lambda}^{\varepsilon}-\eta_{\varepsilon} \phi_{\lambda}^{\varepsilon}\right\|_{L^{2}\left(\Omega_{\varepsilon}\right)} \leq C \varepsilon^{\frac{d(\tilde{p}-2)}{2 \tilde{p}}} .
$$

Here, $\widetilde{p}>2$ is from (3.8) and $C$ depends on $\left|\Omega_{0}\right|,\left\|f_{N}\right\|_{L^{\tilde{p}}(N)}, \max _{i, j, \alpha, \beta}\left\|a_{i j}^{\alpha \beta}\right\|_{L^{\infty}\left(\Omega_{\varepsilon}\right)}, n, m, M$, $\max \{|a|,|b|\}, B$, and the constants from (2.13). Moreover, $C$ is independent of $\varepsilon$. 
Proof. Hölder's inequality and Poincaré's inequality (2.3) imply

$$
\begin{aligned}
\left\|\phi_{\lambda}^{\varepsilon}-\eta_{\varepsilon} \phi_{\lambda}^{\varepsilon}\right\|_{L^{2}\left(\Omega_{\varepsilon}\right)}^{2} & =\int_{\Omega_{\varepsilon}}\left(1-\eta_{\varepsilon}\right)^{2}\left|\phi_{\lambda}^{\varepsilon}\right|^{2} \mathrm{~d} y=\int_{T_{\varepsilon} \cup S_{\varepsilon}^{1} \cup S_{\varepsilon}^{2}}\left(1-\eta_{\varepsilon}\right)^{2}\left|\phi_{\lambda}^{\varepsilon}\right|^{2} \mathrm{~d} y \\
& \leq C\left\|\nabla \phi_{\lambda}^{\varepsilon}\right\|_{L^{\tilde{p}}\left(\Omega_{\varepsilon}\right)}^{2}\left|T_{\varepsilon} \cup S_{\varepsilon}^{1} \cup S_{\varepsilon}^{2}\right|^{\tilde{p}-2} \tilde{p}
\end{aligned}
$$

The estimate (4.6) now follows from (4.1) and (2.2).

We also may approximate the scalar product of 2 eigenfunctions as in the next lemma.

Lemma 4.3. If $\phi_{\lambda_{1}}^{\varepsilon}, \phi_{\lambda_{2}}^{\varepsilon} \in \mathscr{B}\left(a, b, B, \varepsilon_{0}\right)$, then

$$
\left\|\phi_{\lambda_{1}}^{\varepsilon} \cdot \phi_{\lambda_{2}}^{\varepsilon}-\left(\eta_{\varepsilon} \phi_{\lambda_{1}}^{\varepsilon}\right) \cdot\left(\eta_{\varepsilon} \phi_{\lambda_{2}}^{\varepsilon}\right)\right\|_{L^{1}\left(\Omega_{\varepsilon}\right)} \leq C \varepsilon^{\frac{d(\tilde{p}-2)}{\tilde{p}}} .
$$

Here, $\widetilde{p}>2$ is from (3.8) and $C$ depends on $\left|\Omega_{0}\right|,\left\|f_{N}\right\|_{L^{\tilde{p}}(N)}, \max _{i, j, \alpha, \beta}\left\|a_{i j}^{\alpha \beta}\right\|_{L^{\infty}\left(\Omega_{\varepsilon}\right)}, n, m, M$, $\max \{|a|,|b|\}, B$, and the constants from (2.13). Moreover, $C$ is independent of $\varepsilon$.

Proof. Hölder's inequality implies

$$
\begin{aligned}
& \left\|\phi_{\lambda_{1}}^{\varepsilon} \cdot \phi_{\lambda_{2}}^{\varepsilon}-\left(\eta_{\varepsilon} \phi_{\lambda_{1}}^{\varepsilon}\right) \cdot\left(\eta_{\varepsilon} \phi_{\lambda_{2}}^{\varepsilon}\right)\right\|_{L^{1}\left(\Omega_{\varepsilon}\right)} \\
= & \int_{\Omega_{\varepsilon}}\left(1-\eta_{\varepsilon}^{2}\right)\left|\phi_{\lambda_{1}}^{\varepsilon} \cdot \phi_{\lambda_{2}}^{\varepsilon}\right| \mathrm{d} y \leq \int_{T_{\varepsilon} \cup S_{\varepsilon}^{1} \cup S_{\varepsilon}^{2}}\left|\phi_{\lambda_{1}}^{\varepsilon}\right|\left|\phi_{\lambda_{2}}^{\varepsilon}\right| \mathrm{d} y \\
\leq & \left\|\phi_{\lambda_{1}}^{\varepsilon}\right\|_{L^{\tilde{p}}\left(\Omega_{\varepsilon}\right)}\left\|\phi_{\lambda_{2}}^{\varepsilon}\right\|_{L^{\tilde{p}}\left(\Omega_{\varepsilon}\right)}\left|T_{\varepsilon} \cup S_{\varepsilon}^{1} \cup S_{\varepsilon}^{2}\right|^{\frac{\tilde{p}-2}{\tilde{p}}} .
\end{aligned}
$$

Now Poincaré's inequality (2.3), (4.1), and (2.2) give the result (4.7).

The next theorem states that eigenvalues, which are Rayleigh quotients of eigenfunctions, may be approximated with Rayleigh quotients of approximated eigenfunctions on $\Omega_{0}$. That is, $\lambda^{\varepsilon}$ acts as an eigenvalue on $\Omega_{0}$ with corresponding eigenfunction $\eta_{\varepsilon} \phi_{\lambda}^{\varepsilon}$.

Theorem 4.1. Given $\phi=\phi_{\lambda}^{\varepsilon} \in \mathscr{B}\left(a, b, B, \varepsilon_{0}\right)$, there exists $q>0$ depending on $n, \widetilde{p}$, and $d$ such that

$$
\left|R_{0}\left(\eta_{\varepsilon} \phi\right)-\lambda^{\varepsilon}\right| \leq C \varepsilon^{q} .
$$

Here, $C$ depends on $\left|\Omega_{0}\right|,\left\|f_{N}\right\|_{L^{\tilde{p}}(N)}, \max _{i, j, \alpha, \beta}\left\|a_{i j}^{\alpha \beta}\right\|_{L^{\infty}\left(\Omega_{\varepsilon}\right)}, n, m, M, \max \{|a|,|b|\}, B$, and the constants from (2.13). Moreover, $C$ is independent of $\varepsilon$.

Proof. We have

$$
R_{0}\left(\eta_{\varepsilon} \phi\right)-\lambda^{\varepsilon}=\frac{B_{0}\left(\eta_{\varepsilon} \phi, \eta_{\varepsilon} \phi\right)-\int_{N} f_{N} \eta_{\varepsilon} \phi \mathrm{d} \sigma}{\int_{\Omega_{0}} \eta_{\varepsilon}^{2}|\phi|^{2} \mathrm{~d} y}-\frac{B_{\varepsilon}(\phi, \phi)-\int_{\widetilde{N}} f_{N} \phi \mathrm{d} \sigma}{\int_{\Omega_{\varepsilon}}|\phi|^{2} \mathrm{~d} y}
$$




$$
\begin{aligned}
& =R_{0}\left(\eta_{\varepsilon} \phi\right)\left(1-\frac{\int_{\Omega_{0}} \eta_{\varepsilon}^{2}|\phi|^{2} \mathrm{~d} y}{\int_{\Omega_{\varepsilon}}|\phi|^{2} \mathrm{~d} y}\right)+\left(\frac{B_{0}\left(\eta_{\varepsilon} \phi, \eta_{\varepsilon} \phi\right)-B_{\varepsilon}(\phi, \phi)}{\int_{\Omega_{\varepsilon}}|\phi|^{2} \mathrm{~d} y}\right) \\
& +\left(\frac{\int_{\widetilde{N}} f_{N} \phi \mathrm{d} \sigma-\int_{\widetilde{N}} f_{N} \eta_{\varepsilon} \phi \mathrm{d} \sigma}{\int_{\Omega_{\varepsilon}}|\phi|^{2} \mathrm{~d} y}\right) \\
& =: R_{0}\left(\eta_{\varepsilon} \phi\right) \mathrm{I}+\mathrm{II}+\mathrm{III} .
\end{aligned}
$$

Since $I=\left(\int_{\Omega_{\varepsilon}}|\phi|^{2} \mathrm{~d} y-\int_{\Omega_{0}} \eta_{\varepsilon}^{2}|\phi|^{2} \mathrm{~d} y\right) / \int_{\Omega_{\varepsilon}}|\phi|^{2} \mathrm{~d} y$ and $\phi \in \mathscr{B}\left(a, b, B, \varepsilon_{0}\right)$, Lemma $4.3 \mathrm{im}$ plies

$$
|I| \leq C \varepsilon^{\frac{d(\tilde{p}-2)}{\tilde{p}}} .
$$

Following a similar argument from part of the proof of Lemma 4.2 in [17], we obtain

$$
|\mathrm{II}| \leq C \varepsilon^{\frac{d(\tilde{p}-2)}{\tilde{p}}} .
$$

Next from Hölder's inequality, we have

$$
\begin{aligned}
& \int_{\widetilde{N}} f_{N} \phi \mathrm{d} \sigma-\int_{\widetilde{N}} f_{N} \eta_{\varepsilon} \phi \mathrm{d} \sigma \\
\leq & \int_{\widetilde{N}}\left|f_{N}\right||\phi|\left(1-\eta_{\varepsilon}\right) \mathrm{d} \sigma \leq \int_{\widetilde{N} \cap \Delta_{\varepsilon}^{1} \cap \Delta_{\varepsilon}^{2}}\left|f_{N}\right||\phi| \mathrm{d} \sigma \\
\leq & C\left(\int_{\widetilde{N} \cap \Delta_{\varepsilon}^{1} \cap \Delta_{\varepsilon}^{2}}\left|f_{N}\right|^{\widetilde{p}}+|\phi|^{\widetilde{p}} \mathrm{~d} \sigma\right)^{\frac{2}{\tilde{p}}}\left|\widetilde{N} \cap \Delta_{\varepsilon}^{1} \cap \Delta_{\varepsilon}^{2}\right|^{\frac{\tilde{p}-2}{\tilde{p}}} .
\end{aligned}
$$

From the definition of $\Delta_{\varepsilon}^{1}$ and $\Delta_{\varepsilon}^{2}$, we now apply the Poincare estimate (2.4) and use (4.1) to obtain

$$
\int_{\widetilde{N}} f_{N} \phi \mathrm{d} \sigma-\int_{\widetilde{N}} f_{N} \eta_{\varepsilon} \phi \mathrm{d} \sigma \leq C \varepsilon^{\frac{(n-1)(\tilde{p}-2)}{\tilde{p}}} .
$$

Therefore since $\phi \in \mathscr{B}\left(a, b, B, \varepsilon_{0}\right)$,

$$
|\mathrm{III}| \leq C \varepsilon^{\frac{(n-1)(\tilde{p}-2)}{\tilde{p}}}
$$

We also have that

$$
R_{0}\left(\eta_{\varepsilon} \phi\right)=\left(\mathrm{II}+\mathrm{III}+\lambda^{\varepsilon}\right) \frac{\int_{\Omega_{0}}|\phi|^{2}}{\int_{\Omega_{\varepsilon}} \eta_{\varepsilon}^{2}|\phi|^{2} \mathrm{~d} y} .
$$


Thus if we choose $\varepsilon$ so that $C \varepsilon^{\frac{d(\tilde{p}-2)}{\tilde{p}}} \leq \frac{1}{2 B}$, we may apply (4.7) to achieve

$$
\left|R_{0}\left(\eta_{\varepsilon} \phi\right)\right| \leq C\left(\varepsilon^{\frac{d(\tilde{p}-2)}{\tilde{p}}}+\varepsilon^{\frac{(n-1)(\tilde{p}-2)}{\tilde{p}}}+\max \{|a|,|b|\}\right) 2 B^{2}
$$

It follows that (4.9)-(4.13) now give the estimate (4.8).

The next theorem says that $\eta_{\varepsilon} \phi_{\lambda}^{\varepsilon}$ acts as an eigenfunction on $\Omega_{0}$ with corresponding eigenvalue $\lambda^{\varepsilon}$.

Theorem 4.2. Given $\phi=\phi_{\lambda}^{\varepsilon} \in \mathscr{B}\left(a, b, B, \varepsilon_{0}\right)$ and $w \in\left(W_{D}^{1,2}\left(\Omega_{0}\right)\right)^{m}$, we have that

$$
\begin{aligned}
& \quad\left|\int_{\Omega_{0}} a_{i j}^{\alpha \beta}\left(\eta_{\varepsilon} \phi\right)_{i}^{\alpha} w_{j}^{\beta} \mathrm{d} y-\lambda^{\varepsilon} \int_{\Omega_{0}}\left(\eta_{\varepsilon} \phi\right)^{\alpha} w^{\alpha} \mathrm{d} y-\int_{N} f_{N} w \mathrm{~d} \sigma\right| \\
& \leq C \varepsilon^{\frac{(n-1)(\tilde{p}-2)}{2 \tilde{p}}}\|w\|_{\left(W_{D}^{1,2}\left(\Omega_{0}\right)\right)^{m}} .
\end{aligned}
$$

Here, $C$ depends on $\left|\Omega_{0}\right|,\left\|f_{N}\right\|_{L^{\tilde{p}}(N)}, \max _{i, j, \alpha, \beta}\left\|a_{i j}^{\alpha \beta}\right\|_{L^{\infty}\left(\Omega_{\varepsilon}\right)}, n, m, M, \max \{|a|,|b|\}, B$, and the constants from (2.13). Moreover, $C$ is independent of $\varepsilon$.

Proof. We first note that since $\Omega_{0}$ is Lipschitz, we may extend $w$ to $E w$ by even reflection into $\Omega_{\varepsilon}$ such that $\|E w\|_{\left(W_{\tilde{D}}^{1,2}\left(\Omega_{\varepsilon}\right)\right)^{m}} \leq C\|w\|_{\left(W_{D}^{1,2}\left(\Omega_{0}\right)\right)^{m}}$. Such an extension is discussed in [20, Appendix A]. With this in mind, we have

$$
\begin{aligned}
& \quad\left|\int_{\Omega_{0}} a_{i j}^{\alpha \beta}\left(\eta_{\varepsilon} \phi\right)_{i}^{\alpha} w_{j}^{\beta} \mathrm{d} y-\lambda^{\varepsilon} \int_{\Omega_{0}}\left(\eta_{\varepsilon} \phi\right)^{\alpha} w^{\alpha} \mathrm{d} y-\int_{N} f_{N} w \mathrm{~d} \sigma\right| \\
& \leq\left|\int_{\Omega_{0}} a_{i j}^{\alpha \beta}\left[\left(\eta_{\varepsilon}\right)_{i} \phi^{\alpha} w_{j}^{\beta}-\left(\eta_{\varepsilon}\right)_{j} \phi^{\alpha} w^{\beta}\right] \mathrm{d} y\right| \\
& \quad+\left|\int_{\Omega_{\varepsilon}} a_{i j}^{\alpha \beta} \phi_{i}^{\alpha}\left(\eta_{\varepsilon} E w\right)_{j}^{\beta} \mathrm{d} y-\lambda^{\varepsilon} \int_{\Omega_{\varepsilon}} \phi^{\alpha}\left(\eta_{\varepsilon} E w\right)^{\alpha} \mathrm{d} y-\int_{\widetilde{N}} f_{N} \eta_{\varepsilon} E w \mathrm{~d} \sigma\right| \\
& \quad+\left|\int_{\widetilde{N}} f_{N} \eta_{\varepsilon} w \mathrm{~d} \sigma-\int_{N} f_{N} w \mathrm{~d} \sigma\right| \\
& =:|\mathrm{I}|+|\mathrm{II}|+|\mathrm{III}| .
\end{aligned}
$$

First, we may use (2.7), Hölder's inequality, and (4.1) to get

$$
\begin{aligned}
|\mathrm{I}| & \leq \frac{C}{\varepsilon}\|\phi\|_{L^{2}\left(S_{\varepsilon}^{1} \cup S_{\varepsilon}^{2}\right)}\left(\|\nabla w\|_{L^{2}\left(S_{\varepsilon}^{1} \cup S_{\varepsilon}^{2}\right)}+\|w\|_{L^{2}\left(S_{\varepsilon}^{1} \cup S_{\varepsilon}^{2}\right)}\right) \\
& \leq C \varepsilon^{\frac{n(\tilde{p}-2)}{2 \tilde{p}}}\|\nabla \phi\|_{L^{\tilde{p}}\left(\Omega_{\varepsilon}\right)}\|w\|_{\left(W_{D}^{1,2}\left(\Omega_{0}\right)\right)^{m}} \\
& \leq C \varepsilon^{\frac{n(\tilde{p}-2)}{2 \tilde{p}}}\|w\|_{\left(W_{D}^{1,2}\left(\Omega_{0}\right)\right)^{m}} .
\end{aligned}
$$


Also, since $\phi$ solves $L \phi=\lambda^{\varepsilon} \phi$,

$$
\mathrm{II}=0 .
$$

Finally, by Hölder's inequality and the Poincaré estimate (2.4),

$$
\begin{aligned}
\operatorname{III} & \leq \int_{N \cap \Delta_{\varepsilon}^{1} \cap \Delta_{\varepsilon}^{2}}\left|f_{N}\right||w|\left|\eta_{\varepsilon}-1\right| \mathrm{d} \sigma \\
& \leq\left(\int_{N \cap \Delta_{\varepsilon}^{1} \cap \Delta_{\varepsilon}^{2}}\left|f_{N}\right|^{\widetilde{p}} \mathrm{~d} \sigma\right)^{1 / \widetilde{p}}\left(\int_{N \cap \Delta_{\varepsilon}^{1} \cap \Delta_{\varepsilon}^{2}}|w|^{2} \mathrm{~d} \sigma\right)^{1 / 2}\left|N \cap \Delta_{\varepsilon}^{1} \cap \Delta_{\varepsilon}^{2}\right|^{\frac{\tilde{p}-2}{2 \tilde{p}}} \\
& \leq C \varepsilon^{\frac{(n-1)(\widetilde{p}-2)}{2 p}}\|w\|_{\left(W_{D}^{1,2}\left(\Omega_{0}\right)\right)^{m}} .
\end{aligned}
$$

The estimate (4.14) now follows from (4.15)-(4.18).

\section{Conclusion}

This paper adds to study of eigenvalue problems for systems on perturbed domains. Some problems to consider in the future are below:

- Can we study problems with perturbations that have Neumann boundary data if we impose more regularity on the domains?

- If we allow the perturbation to be less general, can we achieve more results?

- Can we allow our domains to satisfy a Robin type boundary condition?

\section{Acknowledgement}

The authors thank the referees for their valuable comments and suggestions in the prepartion of this article.

\section{References}

[1] Babuska I., Výborný R., Continuous dependence of eigenvalues on the domain. Czechoslovak Math. J. 15 (1965), 169-178.

[2] Davies E. B., Eigenvalue stability bounds via weighted Sobolev spaces. Math Z. 214 (2) (1993), 357-371.

[3] Pang M. M. H., Approximation of ground state eigenvalues and eigenfunctions of Dirichlet Laplacians. Bull. Lond. Math. Soc. 29 (1997), 720-730.

[4] Chavel I., Feldman E. A., Spectra of manifolds with small Handles. Comment. Math. Helv. 56 (1) (1981), 83-102.

[5] Anné C., Colbois B., Spectre du Laplacien agissant sur les p-formes différentielles et écrasement d'anses. Math. Ann. 303 (1995), 545-573. 
[6] Daners D., Dirichlet problems on varying domains. J. Differential Equations 188 (2003), 591624.

[7] Burenkov V. I., Lamberti P. D., Sharp spectral stability estimates via the Lebesgue measure of domains for higher order elliptic operators. Rev. Mat. Complut. 25 (2) (2012), 435-457.

[8] Kozlov V., Domain dependence of eigenvalues of elliptic type operators. Math. Ann. 357 (4) (2013), 1509-1539.

[9] Grieser D., Jerison D., Asymptotics of eigenfunctions on plane domains. Pacific J. Math. 240 (1) (2009), 109-133.

[10] Courant R., Hilbert D., Methods of mathematical physics Vol. I, Wiley-Interscience, New York, 1953.

[11] Arrieta J. M., Hale J. K. and Han Q., Eigenvalue problems for nonsmoothly perturbed domains. J. Differential Equations 91 (1991), 24-52.

[12] Jimbo S., Perturbation formula of eigenvalues in a singularly perturbed domain. J. Math. Soc. Japan 45 (2) (1993), 339-356.

[13] Jimbo S., Kosugi S., Spectra of domains with partial degeneration. J. Math. Sci. Univ. Tokyo 16 (2009), 269-414.

[14] Brown R. M., Hislop P. D. and Martinez A., Eigenvalues and resonances for domains with tubes: Neumann boundary conditions. J. Differential Equations 115 (2) (1995), 458-476.

[15] Fang Q., Asymptotic behavior and domain-dependency of solutions to a class of reactiondiffusion systems with large diffusion coefficients. Hiroshima Math. J. 20 (1990), 549-571.

[16] Taylor J. L., Convergence of Dirichlet eigenvalues for elliptic systems on perturbed domains. J. Spectr. Theory 3 (3) (2013), 293-316.

[17] Collins C. D., Taylor J. L., Eigenvalue convergence on perturbed Lipschitz domains for elliptic systems with mixed general decompositions of the boundary. J. Differential Equations $\mathbf{2 6 5}$ (12) (2018), 6187-6209.

[18] Taylor J. L., Ott K. A. and Brown R. M., The mixed problem in Lipschitz domains with general decompositions of the boundary. Trans. Amer. Math. Soc. 365 (2013), 2895-2930.

[19] Cianchi A., Kerman R., and Pick L., Boundary trace inequalities and rearrangements. J. Anal. Math. 105 (1) (2008), 241-265.

[20] Ott K. A., Brown R. M., The mixed problem for the Lamé system in two dimensions. J. Differential Equations 254 (12) (2013), 4373-4400.

[21] Treves F., Basic Linear Partial Differential Equations. Academic Press, New York etc., 1975.

[22] Oleinik O. A., Shamaev A.S., and Yosifian G.A., Mathematical Problems in Elasticity and Homogenization. Elsevier, Amsterdam, 1992.

[23] Gehring F. W., The $L^{p}$-integrability of the partial derivatives of a quasiconformal mapping. Acta Math. 130 (1973), 265-277.

[24] Giaquinta M., Multiple integrals in the calculus of variations and nonlinear elliptic systems. Princeton Univ. Press, 1983.

[25] Giaquinta M., Modica G., Regularity results for some classes of higher order nonlinear elliptic systems. J. Reine Angew. Math. 311/312 (1979), 145-169.

[26] Miyazaki K., $W^{1, p}$ Regularity of eigenfunctions for the mixed problem with nonhomogeneous Neumann data. Murray State Theses and Dissertations 100 (2018), http:/ / digitalcom -mons.murraystate.edu/etd/100.

[27] Ott K. A., Brown R. M., The mixed problem for the Laplacian in Lipschitz domains. Potential Anal. 38 (2013), 1333-1364. 